\title{
Possible $s$-wave annihilation for MeV dark matter with the 21-cm absorption
}

\author{
Lian-Bao Jia ${ }^{*}$ and $\mathrm{Xu}$ Liao \\ School of Science, Southwest University of Science and Technology, Mianyang 621010, China
}

(Received 5 June 2019; published 12 August 2019)

\begin{abstract}
The CMB observation sets stringent constraints on $\mathrm{MeV}$ dark matter (DM) annihilating into charged states or photons in $s$ waves, and the recent observation of the 21-cm absorption at the cosmic dawn reported by EDGES is also very strict for $s$-wave annihilations of MeV DM. The millicharged DM with $p$-wave-dominant annihilations during the freeze-out period is considered in the literature to give an explanation about the $21-\mathrm{cm}$ absorption, with photon-mediated scattering cooling the hydrogen. In this paper, we focus on the annihilation of millicharged DM being $s$-wave dominant. To explain the $21-\mathrm{cm}$ absorption and meanwhile be compatible with the $\mathrm{CMB}$ and 21-cm absorption bounds on DM annihilations, we consider the annihilation close to the resonance, with the new mediator (here a dark photon) mass being slightly above twice the millicharged DM mass. In this case, the annihilation cross section at the temperature $T \rightarrow 0$ could be much smaller than that at $T_{f}$, which would be tolerated by the bounds on DM annihilations, avoiding the excess heating from DM $s$-wave annihilations to the hydrogen gas. The beam dump and lepton collider experiments can be employed to hunt for millicharged DM via the production of the invisible dark photon.
\end{abstract}

DOI: 10.1103/PhysRevD.100.035012

\section{INTRODUCTION}

For dark matter (DM) particles with masses in the range of ten $\mathrm{MeV}$ to hundreds of $\mathrm{TeV}$, the relic abundance of $\mathrm{DM}$ can be obtained via the thermal freeze-out of DM. One DM candidate extensively concerned is weakly interacting massive particles (WIMPs) with masses in the $\mathrm{GeV}-\mathrm{TeV}$ scale, and results from recent DM direct detections [1-8] set stringent constraints on WIMP-nucleon scatterings. In the case of DM being lighter and in the MeV scale, the MeV DM could evade the DM-target nucleus scattering hunters. Thus, the MeV DM is of our concern.

The bulk of cosmological matter density (about $84 \%$ ) is contributed by DM [9], and the typical annihilation cross section of DM during the freeze-out period is about $3 \times 10^{-26} \mathrm{~cm}^{3} / \mathrm{s}$. Furthermore, the cosmic microwave background $(\mathrm{CMB})$ observations at the recombination epoch set upper limits on $s$-wave annihilations of $\mathrm{MeV}$ DM with the annihilation products being of charged states or photons [9,10], which are significantly below the annihilation cross section required by the relic abundance of DM. In addition, the constraint from the recent observation of the $21-\mathrm{cm}$ absorption [11] at the cosmic dawn is

\footnotetext{
*jialb@mail.nankai.edu.cn
}

Published by the American Physical Society under the terms of the Creative Commons Attribution 4.0 International license. Further distribution of this work must maintain attribution to the author(s) and the published article's title, journal citation, and DOI. Funded by SCOAP. also very strict for the $s$-wave annihilation of $\mathrm{MeV} \mathrm{DM}$ [12-14], as the energy injection from DM $s$-wave annihilations would heat the hydrogen gas. Therefore, the $\mathrm{MeV}$ DM with $p$-wave-dominant annihilations during the freezeout period is generally considered in the literature [15-17].

Here, we will focus on the $21-\mathrm{cm}$ absorption. The enhanced 21-cm absorption observed by the EDGES Collaboration [11] indicates that neutral hydrogen at the cosmic dawn would be colder than expected, and a feasible mechanism is that the hydrogen is cooled by the scattering with $\mathrm{MeV}$ millicharged $\mathrm{DM},{ }^{1}$ with photons being the mediator in the scattering [24-29]. ${ }^{2}$ For the 21-cm brightness temperature $T_{21}=-300 \mathrm{mK}$ (the upper limit from EDGES), the required $\mathrm{MeV}$ millicharged DM is in a mass range of about $10-35 \mathrm{MeV}$, which carries a millicharge $\eta e$ with $\eta \sim 5 \times 10^{-6}-5 \times 10^{-5}$, and the millicharged $\mathrm{DM}$ makes up a small fraction $f_{\mathrm{DM}}$ of the total DM relic density [14,36-40], i.e., [Mass of millicharged DM $(\mathrm{MeV}) / 10] \times$ $0.115 \% \lesssim f_{\text {DM }} \lesssim 0.4 \%$.

A large annihilation cross section mediated by new interactions during the freeze-out period is needed to obtain the small fraction of millicharged DM. To explain the 21-cm anomaly, and meanwhile, to avoid constraints from the CMB and 21-cm absorption on $s$-wave annihilations, the $p$-wave-dominant millicharged DM annihilations during

\footnotetext{
${ }^{1}$ A millicharge may be from a kinetic mixing of an extra massless gauge boson [18], or from other scenarios; see, e.g., Refs. [19-23].

${ }^{2}$ See Refs. [30-35] for more about the 21-cm absorption.
} 
the freeze-out period are considered in Refs. [36,41,42]. Is it possible to explain the 21-cm anomaly with the millicharged DM, which, being $s$-wave dominant, annihilates during the freeze-out period? Maybe some extraordinary annihilation mechanism could do the job.

For DM $s$-wave annihilations at the temperature $T \rightarrow 0$, if twice the DM mass were around the mediator mass, the resonant DM annihilation at $T \rightarrow 0$ would be different from that at the freeze-out period [43-45]. Generally, for the mediator mass being slightly below twice the DM mass, the annihilation cross section of DM at $T \rightarrow 0$ could be larger than that at the freeze-out temperature $T_{f}$; for the mediator mass being slightly above twice the DM mass, the annihilation cross section of DM at $T \rightarrow 0$ could be smaller than that at $T=T_{f}$. In the case of the new mediator mass being slightly above twice the millicharged DM mass, the millicharged DM with $s$-wave-dominant annihilations may cause the $21-\mathrm{cm}$ anomaly and meanwhile evade constraints from the CMB and 21-cm absorption. This will be investigated in this paper.

\section{ANNIHILATIONS OF MILLICHARGED DM}

Which kinds of new interactions are needed to obtain the small fraction of millicharged DM is an open question. Here we consider the fermionic millicharged DM with a dark photon as the new mediator, and now the two mediators are the photon and dark photon. The scenario is that the small fraction of millicharged DM is due to darkphoton-mediated $s$-wave annihilations during the freezeout period, and the 21-cm absorption at the cosmic dawn is caused by photon-mediated scattering between millicharged DM and hydrogen. Furthermore, we should keep in mind that there may be more particles in the dark sector, and we focus on the particles that play key roles in transitions or interactions between millicharged DM and ordinary matter.

Besides the fermionic millicharged DM carrying a millicharge $\eta e$, here the DM is also dark charged, and a dark-photon field $\hat{A}^{\prime}$ mediates dark electromagnetism in the dark sector. The dark photon-photon kinetic mixing $\frac{1}{2} \varepsilon \hat{F}_{\mu \nu} \hat{F}^{\prime \mu \nu}$ (see, e.g., Refs. [18,46-52] for more) bridges new transitions between millicharged DM and the standard model (SM) particles, with the field strengths $\hat{F}$ and $\hat{F}^{\prime}$ corresponding to the electromagnetism field $\hat{A}$ and dark electromagnetism field $\hat{A}^{\prime}$, respectively. The mass of the dark photon can be obtained via a Higgs-like mechanism or Stueckelberg mechanism [53]. After diagonalizing the kinetic mixing with the transformation $\hat{A} \rightarrow A+\varepsilon A^{\prime}$, $\hat{A}^{\prime} \rightarrow A^{\prime}$, the physical eigenstate of the dark photon $A^{\prime}$ couples to SM charged fermions:

$$
\mathcal{L}_{i}^{\mathrm{SM}}=-e \varepsilon A_{\mu}^{\prime} J_{\mathrm{em}}^{\mu},
$$

where $J_{\mathrm{em}}^{\mu}$ is the electromagnetic current. In addition, $A^{\prime}$ couples to the fermionic millicharged DM $\chi$ in forms of $-e_{D} A_{\mu}^{\prime} \bar{\chi} \gamma^{\mu} \chi$, where $e_{D}$ is the dark charge.

For fermionic millicharged DM, the annihilation $\bar{\chi} \chi \rightarrow$ $A^{\prime} \rightarrow$ SM mediated by dark photon $A^{\prime}$ is an $s$-wave process, which could be dominant during DM freeze-out. To be able to significantly lower the $s$-wave annihilation of millicharged DM at low temperatures after DM freeze-out, here we consider the case in which the mass of the dark photon is slightly above twice the millicharged DM mass. For teens of $\mathrm{MeV}$ millicharged DM indicated by the $21-\mathrm{cm}$ absorption, the main annihilation products in SM are $e^{+} e^{-}$, and the annihilation cross section is about

$$
\sigma_{1} v_{r} \simeq \frac{1}{2} \frac{\alpha e_{D}^{2} \varepsilon^{2}\left[s\left(m_{\chi}^{2}+m_{e}^{2}\right)+\frac{s^{3}}{4 m_{A^{\prime}}^{2}}\right]\left(1-\frac{4 m_{e}^{2}}{s}\right)^{\frac{1}{2}}}{\left(s-2 m_{\chi}^{2}\right)\left[\left(s-m_{A^{\prime}}^{2}\right)^{2}+m_{A^{\prime}}^{2} \Gamma_{A^{\prime}}^{2}\right]}
$$

where $v_{r}$ is the relative velocity of the two DM particles, the factor $\frac{1}{2}$ is for the required $\bar{\chi} \chi$ pair in DM annihilations, and $s$ is the total invariant mass squared. The width $\Gamma_{A^{\prime}}$ is mainly from $A^{\prime} \rightarrow \bar{\chi} \chi$, with

$$
\Gamma_{A^{\prime}} \approx \frac{e_{D}^{2}\left(m_{A^{\prime}}^{2}-m_{\chi}^{2}\right)}{6 \pi m_{A^{\prime}}}\left(1-\frac{4 m_{\chi}^{2}}{m_{A^{\prime}}^{2}}\right)^{\frac{1}{2}}
$$

The relic density of millicharged $\mathrm{DM} f_{\mathrm{DM}} \Omega_{D} h^{2}\left(\Omega_{D} h^{2}\right.$ is the total relic density of $\mathrm{DM}$, and $f_{\mathrm{DM}}$ is the fraction of millicharged DM) is set by the thermally averaged annihilation cross section $\left\langle\sigma_{1} v_{r}\right\rangle$ via the relation $[54,55]$

$$
f_{\mathrm{DM}} \Omega_{D} h^{2} \simeq \frac{1.07 \times 10^{9} \mathrm{GeV}^{-1}}{m_{\mathrm{Pl}} J_{\mathrm{ann}} \sqrt{g_{*}}},
$$

with

$$
J_{\mathrm{ann}}=\int_{x_{f}}^{\infty} \frac{\left\langle\sigma_{1} v_{r}\right\rangle}{x^{2}} \mathrm{~d} x
$$

The parameter $x=m_{\chi} / T$, and $x_{f}=m_{\chi} / T_{f}$ at the freezeout temperature $T_{f}$ (see, e.g., Ref. [54] for the calculation of $T_{f}$ ). For a pair of DM particles annihilating at $T$ (here $T \ll m_{\chi}$ ), the thermally averaged annihilation cross section can be obtained with methods derived in Ref. [55]. The value of $x_{f} J_{\text {ann }}$ is a typical annihilation cross section related to the relic abundance of millicharged DM.

For the temperature of DM $T \rightarrow 0$ ( $T$ compared with DM mass), the corresponding annihilation cross section of DM mediated by $A^{\prime}$ is different from that at the DM freeze-out period. For $\bar{\chi} \chi \rightarrow e^{+} e^{-}$at $T \rightarrow 0$, contributions from $A^{\prime}$ and the photon are considered, and the annihilation cross section is 


$$
\begin{aligned}
\sigma_{2} v_{r} \simeq & \frac{1}{2} \frac{1}{2 \pi}\left[\left(2 m_{\chi}^{2}+m_{e}^{2}\right)(A+B)(A+B)^{*}\right. \\
& \left.+m_{\chi}^{2}\left(\frac{4 m_{\chi}^{2}}{m_{A^{\prime}}^{2}}-1\right)\left(A A^{*}+2 B \operatorname{Re} A\right)\right]\left(1-\frac{m_{e}^{2}}{m_{\chi}^{2}}\right)^{\frac{1}{2}},
\end{aligned}
$$

where $A, B$ are

$$
A=\frac{e_{D^{\varepsilon}} \varepsilon}{4 m_{\chi}^{2}-m_{A^{\prime}}^{2}+i m_{A^{\prime}} \Gamma_{A^{\prime}}}, \quad B=\frac{\alpha \eta \pi}{m_{\chi}^{2}} .
$$

In addition, the $s$-wave annihilation mode $\bar{\chi} \chi \rightarrow \gamma \gamma$ is deeply suppressed by $\eta^{4}$.

\section{NUMERICAL ANALYSIS}

The millicharged DM is colder than hydrogen at the cosmic dawn. To cool the hydrogen and produce the anomalous $21-\mathrm{cm}$ absorption via photon-mediated scatterings between millicharged DM and hydrogens, the parameter ranges for millicharged DM are as follows: the mass $m_{\chi} \sim 10-35 \mathrm{MeV}$, the millicharge $\eta e$ with $\eta \sim$ $5 \times 10^{-6}-5 \times 10^{-5}$, and the relic fraction $\left[m_{\chi}(\mathrm{MeV}) / 10\right] \times$ $0.115 \% \lesssim f_{\mathrm{DM}} \lesssim 0.4 \%$, as given by the Introduction. In the early Universe, for $m_{\chi} \sim 10 \mathrm{MeV}$, the energy injection from $\bar{\chi} \chi$ annihilations would heat the electron-photon plasma after the electron-neutrino decoupling, and this could lower the effective number of relativistic neutrinos $N_{\text {eff }}$. For Dirac fermionic DM, the relation between $N_{\text {eff }}$ and $m_{\chi} / T_{d}$ was analyzed in Ref. [56], with $T_{d}$ being the neutrino decoupling temperature. Considering the Planck 2018 results [9] $N_{\text {eff }}=2.99 \pm 0.17$, we have $m_{\chi} / T_{d} \gtrsim$ 5.56 , with $N_{\text {eff }} \gtrsim 2.82$ adopted. Taking $T_{d} \gtrsim 2 \mathrm{MeV}$, we have $m_{\chi} \gtrsim 11.1 \mathrm{MeV}$. Thus, the mass range of fermionic millicharged DM is $11.1 \lesssim m_{\chi} \lesssim 35 \mathrm{MeV}$.

In the dark ages, the energy injection from $s$-wave annihilations of millicharged DM could induce excess heating to the hydrogen gas, and thus the anomalous $21-\mathrm{cm}$ absorption sets stringent constraints on $s$-wave annihilations of millicharged DM. For the annihilation $\bar{\chi} \chi \rightarrow$ $e^{+} e^{-}$at $T \rightarrow 0$, if the matter temperature $T_{m}<4 \mathrm{~K}$ is chosen at redshift $z=17.2$, the corresponding annihilation cross section is $\lesssim 10^{-26}-10^{-25} \mathrm{~cm}^{3} / \mathrm{s}$, with $m_{\chi} \sim 10-35 \mathrm{MeV}$ and $f_{\mathrm{DM}}=0.01$ [14]. Thus, to cool the hydrogen and avoid excess heating, the weighted annihilation cross section of $f_{\text {DM }}^{2} \times$ [annihilation cross section] at $T \rightarrow 0$ should be $\lesssim 10^{-30} \mathrm{~cm}^{3} / \mathrm{s}$. Here the $s$-wave annihilation $\bar{\chi} \chi \rightarrow A^{\prime} \rightarrow$ $e^{+} e^{-}$is dominant during millicharged DM freeze-out. To escape constraints from CMB and the 21-cm absorption on this $s$-wave annihilation, we consider the case that the mass $m_{A^{\prime}}$ is sightly above $2 m_{\chi}$. Note $\xi=m_{A^{\prime}} / 2 m_{\chi}$, and here $\xi$ is slightly above 1 . In this case, the thermally averaged annihilation cross section at temperature $T \rightarrow 0$ could be smaller than that at $T=T_{f}$. Take $m_{\chi}=20 \mathrm{MeV}$,
$f_{\mathrm{DM}}=0.4 \%, e_{D}=0.1$, and $\xi=1.1$ as an example to evaluate the temperature-dependent annihilation cross section $\left\langle\sigma_{1} v_{r}\right\rangle_{T}$ with $x\left(x=m_{\chi} / T\right)$, and the result is shown in Fig. 1. It can be seen that the corresponding annihilation cross section $\left\langle\sigma_{1} v_{r}\right\rangle_{0}$ of millicharged $\mathrm{DM}$ at $T \rightarrow 0$ is smaller than $\left\langle\sigma_{1} v_{r}\right\rangle_{T_{f}}$ at the freeze-out period $T=T_{f}$. To further manifest the resonance effect for different $\xi$, we take $m_{\chi}=20 \mathrm{MeV}, m_{\chi} / T_{f}=22,24$, and $\Gamma_{A^{\prime}} / m_{A^{\prime}}=1 \times$ $10^{-3}, 3 \times 10^{-4}$ as an example to evaluate the ratio of $\left\langle\sigma_{1} v_{r}\right\rangle_{0} /\left\langle\sigma_{1} v_{r}\right\rangle_{T_{f}}$ with $\xi$, and the result is depicted in Fig. 2. It can be seen that the $s$-wave annihilation $\bar{\chi} \chi \rightarrow$ $A^{\prime} \rightarrow e^{+} e^{-}$at $T \rightarrow 0$ could be much smaller than that at $T=T_{f}$; e.g., for $0.02 \lesssim \xi-1 \lesssim 0.13$, the ratio is $\lesssim 10^{-2}$. Thus, for millicharged $\mathrm{DM}$ in $\mathrm{MeV}$ scale, the $s$-wavedominant DM annihilation during the freeze-out period may be allowed by constraints from CMB and the $21-\mathrm{cm}$ absorption, and this will be further analyzed in the following.

The annihilation cross section of millicharged DM at the freeze-out period is set by the relic density of millicharged $\mathrm{DM} f_{\mathrm{DM}} \Omega_{D} h^{2}$, with $\Omega_{D} h^{2}=0.120 \pm 0.001$ [9]. For $\bar{\chi} \chi \rightarrow$ $e^{+} e^{-}$at $T \rightarrow 0$, suppose the upper limit of the weighted annihilation cross section $f_{\mathrm{DM}}^{2}\left\langle\sigma_{2} v_{r}\right\rangle_{0}$ (corresponding to the case of $f_{\mathrm{DM}} \sim 0.004$ and $\eta \sim 5 \times 10^{-5}$ ) is tolerated by constraints from $\mathrm{CMB}$ and the anomalous $21-\mathrm{cm}$ absorption, and the range of $\xi\left(\xi=m_{A^{\prime}} / 2 m_{\chi}\right)$ allowed can be derived for a given value of $e_{D}$ (here $e_{D}=0.1$ is taken), as depicted in Fig. 3. It can be seen that, though constraints of the weighted annihilation cross section $\lesssim 10^{-30} \mathrm{~cm}^{3} / \mathrm{s}$ from the anomalous $21-\mathrm{cm}$ absorption are very strict for additional energy injection from $s$-wave annihilations of millicharged DM, the $s$-wave-dominant millicharged DM annihilations with $0.004 \lesssim \xi-1 \lesssim 0.085$ can be compatible with the anomalous $21-\mathrm{cm}$ absorption. Thus, the millicharged DM with $s$-wave-dominant annihilations could cool the hydrogen and induce the anomalous

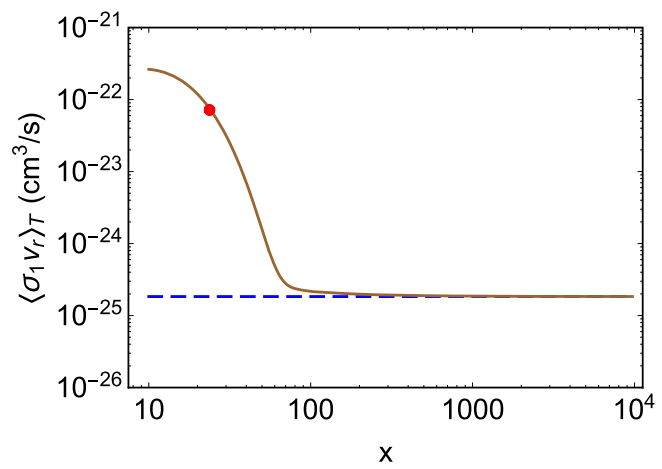

FIG. 1. The temperature-dependent annihilation cross section $\left\langle\sigma_{1} v_{r}\right\rangle_{T}$ as a function of $x$, with $x=m_{\chi} / T$ and $m_{\chi}=20 \mathrm{MeV}$. The solid curve is for the case of $f_{\mathrm{DM}}=0.4 \%, e_{D}=0.1$, and $\xi=1.1$ (where $\xi=m_{A^{\prime}} / 2 m_{\chi}$ ). The dot is the annihilation cross section $\left\langle\sigma_{1} v_{r}\right\rangle_{T_{f}}$ at $T=T_{f}\left(x_{f} \simeq 23.65\right)$. For comparison, the dashed line is the result of $\left\langle\sigma_{1} v_{r}\right\rangle_{0}$ at $T \rightarrow 0$. 


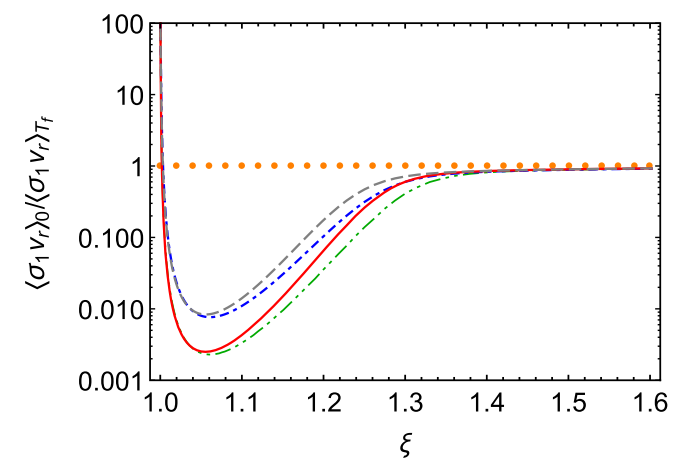

FIG. 2. The ratio $\left\langle\sigma_{1} v_{r}\right\rangle_{0} /\left\langle\sigma_{1} v_{r}\right\rangle_{T_{f}}$ as a function of $\xi$ $\left(\xi=m_{A^{\prime}} / 2 m_{\chi}\right)$, with $m_{\chi}=20 \mathrm{MeV}$. Here the dashed curve is for the case of $\Gamma_{A^{\prime}} / m_{A^{\prime}}=1 \times 10^{-3}$ and $x_{f}=24$ (where $\left.x_{f}=m_{\chi} / T_{f}\right)$, the dot-dashed curve is for the case of $\Gamma_{A^{\prime}} / m_{A^{\prime}}=$ $1 \times 10^{-3}$ and $x_{f}=22$, the solid curve is for the case of $\Gamma_{A^{\prime}} / m_{A^{\prime}}=3 \times 10^{-4}$ and $x_{f}=24$, and the dot-dot-dashed curve is for the case of $\Gamma_{A^{\prime}} / m_{A^{\prime}}=3 \times 10^{-4}$ and $x_{f}=22$. For comparison, the dotted line is for the ratio being equal to 1 .

21-cm absorption at the cosmic dawn, and meanwhile avoid excessive energy injection from $s$-wave annihilations which would cause excess heating to the hydrogen.

The dark photon mainly decays into $\bar{\chi} \chi$, and this invisible decay could be produced at lepton collider and beam dump experiments [57-61], or the kinetic mixing parameter $\varepsilon$

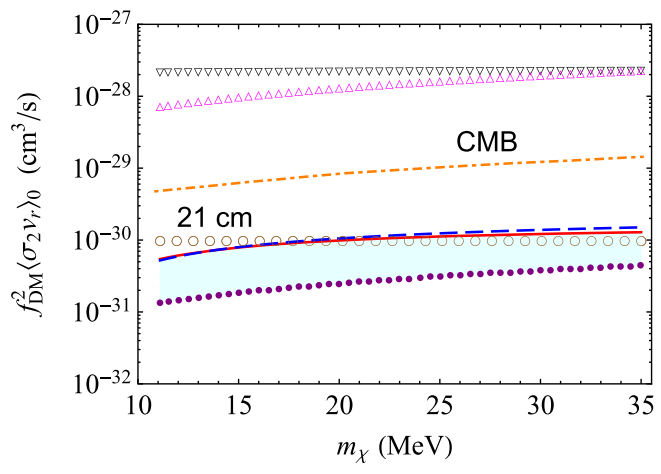

FIG. 3. The weighted annihilation cross section $f_{\mathrm{DM}}^{2}\left\langle\sigma_{2} v_{r}\right\rangle_{0}$ for given values of $\xi\left(\xi=m_{A^{\prime}} / 2 m_{\chi}\right)$, with $e_{D}=0.1$ and $m_{\chi}$ in a range of $11.1-35 \mathrm{MeV}$. The band is the range of $f_{\mathrm{DM}}^{2}\left\langle\sigma_{2} v_{r}\right\rangle_{0}$, with the parameters $0.004 \lesssim \xi-1 \lesssim 0.085,\left[m_{\chi}(\mathrm{MeV}) / 10\right] \times$ $0.115 \% \lesssim f_{\text {DM }} \lesssim 0.4 \%$, and $5 \times 10^{-6} \lesssim \eta \lesssim 5 \times 10^{-5}$. The upper limit of the band corresponds to $f_{\mathrm{DM}}=0.4 \%, \eta=5 \times 10^{-5}$, and $\xi=1.004$ (the solid curve) or $\xi=1.085$ (the dashed curve). The solid dotted curve is the lower bound, with $f_{\mathrm{DM}}=$ $\left[m_{\chi}(\mathrm{MeV}) / 10\right] \times 0.115 \%, \eta=5 \times 10^{-6}$, and $\xi \simeq 1.0242$. The dot-dashed curve and empty dotted curve are constraints from the CMB observation [10] and the anomalous 21-cm absorption with $T_{m}<4 \mathrm{~K}$ at $z=17.2$ [14], respectively. For comparison, the triangle and reverse triangle curves are the weighted typical annihilation cross section $f_{\mathrm{DM}}^{2} x_{f} J_{\text {ann }}$ (which is not sensitive to the resonance effect) required to obtain the relic fractions $f_{\mathrm{DM}}=$ $\left[m_{\chi}(\mathrm{MeV}) / 10\right] \times 0.115 \%$ and $f_{\mathrm{DM}}=0.4 \%$, respectively. would be restricted by experiments. For a given $\xi$ $\left(\xi=m_{A^{\prime}} / 2 m_{\chi}\right)$, to obtain the small fraction $f_{\mathrm{DM}}$ of millicharged DM, the range of $\varepsilon$ is derived with $\xi=1.085$, 1.004 , and $e_{D}=0.1$, as shown in Fig. 4. It can be seen that the range of $\varepsilon$ indicated by the $21-\mathrm{cm}$ absorption is allowed by recent lepton collision experiments, such as $B A B A R$ [57] and NA64 [58,59]. The dark photon can be further investigated at future experiments, such as NA64 [59], Belle II [63], and the Light Dark Matter eXperiment (LDMX) [64].

Now we give a brief discussion about the detection of millicharged DM at underground experiments. For the millicharged DM of concern, magnetic fields in the Milky Way could expel most of the millicharged DM from the Galactic disk, as estimated in Refs. [24,65,66]. Even though a small amount of millicharged DM would remain in the Galactic disk, the magnetic fields related to the solar wind and the Earth's magnetic field would substantially reduce the flux of millicharged DM arriving at the Earth's surface. In addition, for underground experiments, the terrestrial effect of a particle penetrating the Earth and strongly interacting with overburden matter (e.g., photonor dark-photon-mediated large interactions related to the electric charge of nuclei) could deplete the particle's energy and significantly reduce the detection sensitivity $[67,68]$. For the millicharged DM, the reference cross section $\bar{\sigma}_{e}$ (see, e.g., Ref. [69] for more) of $\chi$-electron scattering with a photon as the mediator is in the range $\sim 3.5 \times 10^{-26}-3.5 \times 10^{-24} \mathrm{~cm}^{2}$, and the rock or concrete shielding at depths of $\sim 3-10$ meters could result in little

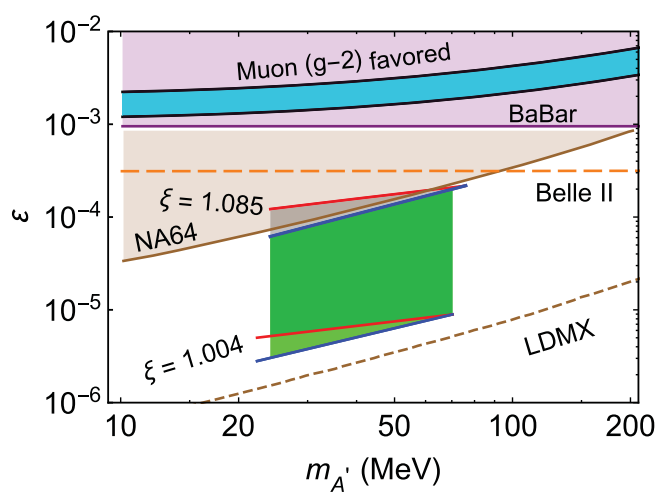

FIG. 4. The value of $\varepsilon$ as a function of $m_{A^{\prime}}$ with $\xi$ $\left(\xi=m_{A^{\prime}} / 2 m_{\chi}\right)=1.085,1.004$ and $e_{D}=0.1$. The bands are ranges of $\varepsilon$ indicated by the $21-\mathrm{cm}$ anomaly. For $\xi=1.085$, the upper red and blue solid curves correspond to $f_{\mathrm{DM}}=$ $\left[m_{\chi}(\mathrm{MeV}) / 10\right] \times 0.115 \%$ and $f_{\mathrm{DM}}=0.4 \%$, respectively. For $\xi=1.004$, the lower red and blue solid curves correspond to $f_{\mathrm{DM}}=\left[m_{\chi}(\mathrm{MeV}) / 10\right] \times 0.115 \%$ and $f_{\mathrm{DM}}=0.4 \%$, respectively. The constraints from BABAR [57] and NA64 [59] and the regions favored by the muon $\mathrm{g}-2$ [62] are annotated in the figure. The upper and lower dashed curves are the expected sensitivities set by $20 \mathrm{fb}^{-1}$ Belle II data [63] and the ultimate reach of LDMX [64], respectively. 
detection signal of millicharged DM [68]. In this case, the millicharged DM of concern will evade constraints from underground experiments, such as XENON10 [70,71], XENON100 [71], and DarkSide-50 [72]. Moreover, the above case may be not the whole thing for the millicharged DM, as analyzed in Ref. [73]. The millicharged DM could be accelerated by supernova shocks, and the evacuation of millicharged DM from the disk may not be effective due to the diffusion of millicharged DM from the halo [73]. Hence, there are uncertainties about the millicharged DM in the disk, and corresponding uncertainties in direct detections.

\section{CONCLUSION AND DISCUSSION}

In this paper, the $s$-wave-dominant annihilations of $\mathrm{MeV}$ millicharged DM has been studied with the anomalous 21-cm absorption. The photon-mediated scattering could cool the hydrogen and induce the $21-\mathrm{cm}$ anomaly at the cosmic dawn, and the required small fraction $f_{\mathrm{DM}}$ of millicharged DM is predominantly contributed by the darkphoton-mediated annihilations during the freeze-out period. For $s$-wave-dominant DM annihilations, to be compatible with stringent constraints from $\mathrm{CMB}$ and the anomalous 21-cm absorption, the annihilation is considered to be close to the resonance, with $\xi\left(\xi=m_{A^{\prime}} / 2 m_{\chi}\right)$ being slightly above 1 . In this case, the annihilation cross section at $T \rightarrow 0$ could be much smaller than that at $T=T_{f}$. For $\xi$ in a range of $0.004 \lesssim \xi-1 \lesssim 0.085\left(e_{D}=0.1\right)$, the weighted annihilation cross section $f_{\mathrm{DM}}^{2}\left\langle\sigma_{2} v_{r}\right\rangle_{0}$ could be $\lesssim 10^{-30} \mathrm{~cm}^{3} / \mathrm{s}$, which is tolerated by constraints from the anomalous $21-\mathrm{cm}$ absorption with $T_{m}<4 \mathrm{~K}(z=17.2)$, avoiding excess heating to the hydrogen.

For millicharged DM with the millicharge $\eta e$, the spatial magnetic fields and the terrestrial effect of large interactions between DM and ordinary matter result in the lowvelocity millicharged DM remained in the disk evading DM direct-detection experiments, while the millicharged DM accelerated by supernova shocks may be detectable. As there are uncertainties about the millicharged DM in the disk, corresponding further explorations of millicharged DM are needed. The beam dump and lepton collider experiments can do the job of hunting for millicharged DM, such as NA64, Belle II, and LDMX, especially for the case of a large $\xi-1$. We look forward to the exploration of millicharged DM at future lepton experiments. In addition, neutrino experiments could also be employed to search for MeV DM [74,75], and the terrestrial effect needs to be taken into account for the investigation of millicharged DM.

\section{ACKNOWLEDGMENTS}

This work was partly supported by the National Natural Science Foundation of China under Contract No. 11505144, and the Longshan Academic Talent Research Supporting Program of SWUST under Contract No. 18LZX415.
[1] R. Agnese et al. (SuperCDMS Collaboration), Phys. Rev. D 97, 022002 (2018).

[2] D. S. Akerib et al. (LUX Collaboration), Phys. Rev. Lett. 118, 021303 (2017).

[3] X. Cui et al. (PandaX-II Collaboration), Phys. Rev. Lett. 119, 181302 (2017).

[4] E. Aprile et al. (XENON Collaboration), Phys. Rev. Lett. 121, 111302 (2018).

[5] D. S. Akerib et al. (LUX Collaboration), Phys. Rev. Lett. 118, 251302 (2017).

[6] J. Xia et al. (PandaX-II Collaboration), Phys. Lett. B 792, 193 (2019).

[7] E. Aprile et al. (XENON Collaboration), Phys. Rev. Lett. 122, 141301 (2019).

[8] C. Amole et al. (PICO Collaboration), Phys. Rev. D 100, 022001 (2019).

[9] N. Aghanim et al. (Planck Collaboration), arXiv:1807 .06209 .

[10] T. R. Slatyer, Phys. Rev. D 93, 023527 (2016).

[11] J. D. Bowman, A. E. E. Rogers, R. A. Monsalve, T. J. Mozdzen, and N. Mahesh, Nature (London) 555, 67 (2018).
[12] G. D’Amico, P. Panci, and A. Strumia, Phys. Rev. Lett. 121, 011103 (2018).

[13] K. Cheung, J. L. Kuo, K. W. Ng, and Y. L. S. Tsai, Phys. Lett. B 789, 137 (2019).

[14] H. Liu and T. R. Slatyer, Phys. Rev. D 98, 023501 (2018).

[15] P. McDonald, R. J. Scherrer, and T. P. Walker, Phys. Rev. D 63, 023001 (2000).

[16] R. Diamanti, L. Lopez-Honorez, O. Mena, S. PalomaresRuiz, and A.C. Vincent, J. Cosmol. Astropart. Phys. 02 (2014) 017.

[17] L. B. Jia and X. Q. Li, Eur. Phys. J. C 76, 706 (2016).

[18] B. Holdom, Phys. Lett. 166B, 196 (1986).

[19] B. Kors and P. Nath, Phys. Lett. B 586, 366 (2004).

[20] D. Feldman, Z. Liu, and P. Nath, Phys. Rev. D 75, 115001 (2007).

[21] K. Cheung and T. C. Yuan, J. High Energy Phys. 03 (2007) 120.

[22] J. M. Cline, Z. Liu, and W. Xue, Phys. Rev. D 85, 101302 (2012).

[23] C. Kouvaris, Phys. Rev. D 88, 015001 (2013).

[24] R. Barkana, Nature (London) 555, 71 (2018). 
[25] W. L. Xu, C. Dvorkin, and A. Chael, Phys. Rev. D 97, 103530 (2018).

[26] J. B. Muñoz and A. Loeb, Nature (London) 557, 684 (2018).

[27] A. Fialkov, R. Barkana, and A. Cohen, Phys. Rev. Lett. 121, 011101 (2018).

[28] M. S. Mahdawi and G. R. Farrar, J. Cosmol. Astropart. Phys. 10 (2018) 007.

[29] K. K. Boddy, V. Gluscevic, V. Poulin, E. D. Kovetz, M. Kamionkowski, and R. Barkana, Phys. Rev. D 98, 123506 (2018).

[30] J. Mirocha and S. R. Furlanetto, Mon. Not. R. Astron. Soc. 483, 1980 (2019).

[31] C. Li and Y. F. Cai, Phys. Lett. B 788, 70 (2019).

[32] C. Feng and G. Holder, Astrophys. J. 858, L17 (2018).

[33] S. Fraser et al., Phys. Lett. B 785, 159 (2018).

[34] M. Pospelov, J. Pradler, J. T. Ruderman, and A. Urbano, Phys. Rev. Lett. 121, 031103 (2018).

[35] A. Widmark, J. Cosmol. Astropart. Phys. 06 (2019) 014.

[36] A. Berlin, D. Hooper, G. Krnjaic, and S. D. McDermott, Phys. Rev. Lett. 121, 011102 (2018).

[37] R. Barkana, N. J. Outmezguine, D. Redigolo, and T. Volansky, Phys. Rev. D 98, 103005 (2018).

[38] T. R. Slatyer and C. L. Wu, Phys. Rev. D 98, 023013 (2018).

[39] J. B. Muñoz, C. Dvorkin, and A. Loeb, Phys. Rev. Lett. 121, 121301 (2018).

[40] E. D. Kovetz, V. Poulin, V. Gluscevic, K. K. Boddy, R. Barkana, and M. Kamionkowski, Phys. Rev. D 98, 103529 (2018).

[41] L. B. Jia, Eur. Phys. J. C 79, 80 (2019).

[42] L. B. Jia, X. J. Deng, and C. F. Liu, Eur. Phys. J. C 78, 956 (2018).

[43] M. Ibe, H. Murayama, and T. T. Yanagida, Phys. Rev. D 79, 095009 (2009).

[44] J. Kozaczuk and T. A. W. Martin, J. High Energy Phys. 04 (2015) 046.

[45] M. Duch and B. Grzadkowski, J. High Energy Phys. 09 (2017) 159.

[46] L. B. Okun, Zh. Eksp. Teor. Fiz. 83, 892 (1982) [Sov. Phys. JETP 56, 502 (1982)].

[47] P. Galison and A. Manohar, Phys. Lett. 136B, 279 (1984).

[48] P. Fayet, Nucl. Phys. B347, 743 (1990).

[49] R. Foot and S. Vagnozzi, Phys. Lett. B 748, 61 (2015).

[50] S. Bilmis, I. Turan, T. M. Aliev, M. Deniz, L. Singh, and H. T. Wong, Phys. Rev. D 92, 033009 (2015).
[51] J. L. Feng, J. Smolinsky, and P. Tanedo, Phys. Rev. D 93, 015014 (2016); 96, 099901(E) (2017).

[52] F. P. Huang and H. S. Lee, Int. J. Mod. Phys. A 34, 1950012 (2019).

[53] E. C. G. Stueckelberg, Helv. Phys. Acta 11, 225 (1938).

[54] K. Griest and D. Seckel, Phys. Rev. D 43, 3191 (1991).

[55] P. Gondolo and G. Gelmini, Nucl. Phys. B360, 145 (1991).

[56] C. M. Ho and R. J. Scherrer, Phys. Rev. D 87, 023505 (2013).

[57] J. P. Lees et al. (BABAR Collaboration), Phys. Rev. Lett. 119, 131804 (2017).

[58] D. Banerjee et al. (NA64 Collaboration), Phys. Rev. D 97, 072002 (2018).

[59] NA64 Collaboration, arXiv:1906.00176.

[60] E. Izaguirre, G. Krnjaic, P. Schuster, and N. Toro, Phys. Rev. D 88, 114015 (2013).

[61] D. Banerjee et al. (NA64 Collaboration), Phys. Rev. Lett. 118, 011802 (2017).

[62] G. W. Bennett et al. (Muon g-2 Collaboration), Phys. Rev. D 73, 072003 (2006).

[63] E. Kou et al. (Belle-II Collaboration), arXiv:1808.10567.

[64] T. Åkesson et al. (LDMX Collaboration), arXiv:1808 .05219 .

[65] L. Chuzhoy and E. W. Kolb, J. Cosmol. Astropart. Phys. 07 (2009) 014.

[66] S. D. McDermott, H. B. Yu, and K. M. Zurek, Phys. Rev. D 83, 063509 (2011).

[67] T. Emken, C. Kouvaris, and I. M. Shoemaker, Phys. Rev. D 96, 015018 (2017).

[68] T. Emken, R. Essig, C. Kouvaris, and M. Sholapurkar, arXiv:1905.06348.

[69] R. Essig, J. Mardon, and T. Volansky, Phys. Rev. D 85, 076007 (2012).

[70] R. Essig, A. Manalaysay, J. Mardon, P. Sorensen, and T. Volansky, Phys. Rev. Lett. 109, 021301 (2012).

[71] R. Essig, T. Volansky, and T. T. Yu, Phys. Rev. D 96, 043017 (2017).

[72] P. Agnes et al. (DarkSide Collaboration), Phys. Rev. Lett. 121, 111303 (2018).

[73] D. Dunsky, L. J. Hall, and K. Harigaya, J. Cosmol. Astropart. Phys. 07 (2019) 015.

[74] S. F. Ge and I. M. Shoemaker, J. High Energy Phys. 11 (2018) 066.

[75] V. De Romeri, K. J. Kelly, and P. A. N. Machado, arXiv: 1903.10505. 